\title{
Agôn
}

Revue des arts de la scène

HS 1 | 2011

Mettre en scène l'événement

\section{Le cours, lieu du détour et du retour}

L'accompagnement pédagogique des lycéens

\section{Marie-Laure Basuyaux}

\section{(2) OpenEdition}

Journals

Édition électronique

URL : http://journals.openedition.org/agon/1794

DOI : 10.4000/agon. 1794

ISSN : 1961-8581

Éditeur

Association Agôn

Référence électronique

Marie-Laure Basuyaux, "Le cours, lieu du détour et du retour », Agôn [En ligne], HS 1 | 2011, mis en ligne le 05 septembre 2011, consulté le 17 avril 2020. URL : http://journals.openedition.org/agon/1794 ; DOI : https://doi.org/10.4000/agon.1794 


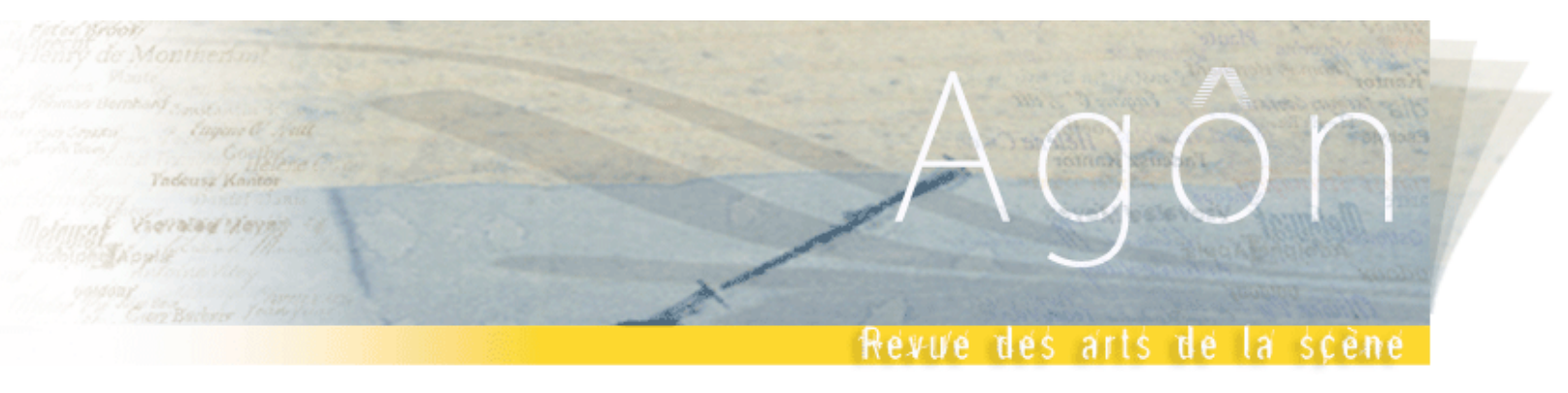

\title{
Le cours, lieu du détour et du retour
}

\section{L'accompagnement pédagogique des lycéens}

\author{
Marie-Laure BASUYAUX
}

Agrégée de Lettres Modernes et docteur en Littérature française (Témoigner clandestinement, Garnier Classiques, 2009), Marie-Laure Basuyaux est professeur au lycée Voillaume d'Aulnay-sous-Bois. Dans ce texte (juillet 2011), elle témoigne du travail d'accompagnement réalisé pendant l'année 2010-2011 avec les lycéens du projet « D'un 11 septembre à l'autre ».

\section{«Ça a débuté comme ça »}

Chacun d'entre nous a vécu « son » début du projet « D'un 11 septembre à l'autre ». Pour moi, ce fut le jour où Arnaud Meunier me proposa, dix ans après notre première collaboration pour un atelier artistique, de faire participer une classe de Première à la création de 11 septembre 2001. Si je me souviens aussi bien du jour où cette proposition nous a été faite, c'est qu'elle a été à la source de deux sentiments bien contradictoires : l'enthousiasme à l'idée de travailler à nouveau avec un metteur en scène et une compagnie dont nous avions régulièrement suivi le travail (notamment pour les mises en scène récentes de King et de Pardessus bord), et l'angoisse à l'idée d'aborder cet événement avec nos élèves, dans un établissement où les questions identitaires et religieuses sont parfois sources de tensions et de crispations. Lors de la première réunion avec les partenaires du projet pour mettre sur pied l'organisation de l'année, nous avons demandé à l'association Citoyenneté Jeunesse de nous apporter l'aide d'un intervenant pour aborder la question du 11 septembre ; nous exagérions à peine en déclarant que ce 11 septembre, nous en cauchemardions désormais toutes les nuits...

Les équipes pédagogiques qui ont décidé de participer à cette aventure étaient bien sûr aguerries à la mise en place de projets culturels et aux collaborations avec des artistes. Ce projet nous a pourtant placés face à des situations et des questions inédites : quels cadres institutionnels pour une entreprise qui ne relève, par son nombre d'heures de répétition, ni de la « Classe à Projet Artistique et Culturel », ni de l' " Atelier artistique » ? Comment articuler les ateliers de pratique théâtrale au temps scolaire dès lors que les répétitions intègrent des week-ends et des périodes de vacances ? Comment passer de l'activité obligatoire à la participation basée sur le volontariat sans perdre l'essentiel des élèves ? Comment permettre une véritable rencontre entre les trois classes participant au projet ? Car contrairement à ce que l'on peut penser, trois classes issues de différents lycées de Seine-Saint-Denis ne se ressemblent pas nécessairement, et, de fait nos classes venues de Bondy, de Noisy-le-Grand et d'Aulnay-sous-Bois sont arrivées avec des identités distinctes, de fortes préventions et la peur d'être jugées par leurs camarades. À les voir s'asseoir systématiquement à distance les uns 
des autres en trois groupes compacts, rien n'indiquait qu'elles finiraient par former cette « compagnie éphémère » que nous appelions de nos vœux.

Le projet a été présenté aux élèves fin septembre. Nous avions certaines inquiétudes - dont nous sourions aujourd'hui - à l'idée de dévoiler aux élèves le titre de la pièce. Quel n'a pas été notre étonnement de découvrir la mollesse de leur première réaction; nous qui comptions travailler à partir de leur mémoire de l'événement, nous avons pu mesurer la minceur de leurs souvenirs : la plupart d'entre eux ne se souvenaient pas, à l'inverse de nous, du moment et de l'endroit où ils avaient appris les attentats, quelques-uns avaient des souvenirs fortement liés à l'enfance : Karima se rappelle n'avoir pas pu regarder ses habituels dessins animés car sa mère regardait les flashs d'information en boucle.

Même déconvenue lors de la première rencontre entre l'équipe artistique du projet et les élèves : la classe a réservé une attention polie à Arnaud Meunier et à la Compagnie de la Mauvaise graine, réserve dont rend bien compte le petit texte de Moussou :

« Me voilà dans cette salle de classe avec tous ces inconnus qui me présentent un projet dont j'ignore encore presque tout. Ils parlent chacun à leur tour en souriant, en espérant me convaincre. Je les écoute parler (...). Mais j'ai l'impression que ça demandera beaucoup de travail et je ne sais pas si j'en serai capable. C'est comme un grand combat intérieur entre ma fainéantise et mon envie de participer à ce projet. Je ne sais pas qui sera le vainqueur ».

Étonnement enfin devant leur version de la théorie du complot, qui n'était pas tout à fait celle à laquelle nous nous attendions : pour une large partie de nos élèves de Première, le 11 septembre est en réalité le fruit d'un complot tramé par les «Illuminatis ». Ce type de certitude nous a fait sentir l'écart qui sépare une génération qui a découvert l'événement en direct sur toutes les télévisions du monde et une génération qui l'a surtout appréhendé à travers des scénarios diffusés sur internet.

\section{Le cours : lieu du détour}

Avouons-le : nous n'avons pas fait à proprement parler de cours sur 11 septembre 2001 : ni sur la pièce (en cours de français), ni sur l'événement (en cours d'histoire). En ce sens, le vrai lieu de la rencontre avec cette pièce de Vinaver a bien été le plateau ${ }^{1}$. Notre approche, à nous enseignants, a davantage relevé de l'accompagnement, de la sensibilisation et d'une forme stratégie du détour.

\footnotetext{
${ }^{1}$ Pour ce qui concerne le lycée Voillaume d'Aulnay-sous-Bois, les 35 élèves de la classe ont participé à des ateliers de pratique théâtrale de trois heures chaque vendredi après-midi d'octobre à janvier, après une immersion inaugurale de trois journées complètes au Forum de Blanc Mesnil en compagnie des classes de Noisy et de Bondy. À partir de janvier, Arnaud Meunier a demandé aux élèves de participer à des week-ends de répétition, à raison d'un week-end par mois environ. La classe s'est alors partagée en deux groupes, l'un poursuivant les ateliers de jeu y compris certains week-ends, l'autre menant diverses activités autour du projet avec l'équipe enseignante, uniquement les vendredis après-midi (travail d'enquêtes, rédaction d'une pièce, rédaction d'articles, enregistrement d'émissions de radio). Les ateliers de jeu ont été menés par les comédiens tandis que les enseignants participaient au même titre que les élèves aux exercices proposés. Les élèves ont répété une semaine complète durant les vacances d'avril, puis une présentation publique du travail a été proposée aux familles. Après cette présentation, les élèves désirant monter sur scène en septembre ont poursuivi jusqu'à mi-mai les ateliers de jeu hebdomadaires et les week-ends de répétition. Les dernières semaines du calendrier scolaire ont été consacrées exclusivement à la préparation du baccalauréat de français, les élèves retrouvant l'équipe artistique à Saint-Étienne pour dix-huit jours de répétition fin août-début septembre. Au total, ils auront bénéficié d'une centaine d'heures d'atelier et de cinq semaines de répétition.
} 
En histoire, le cours sur le 11 septembre viendra en son temps, c'est-à-dire l'année prochaine en Terminale selon la logique proposée par les programmes. Nous avons donc choisi d'aborder cet événement d'une manière indirecte, par le biais d'un travail d'enquête sur la mémoire du 11 septembre ou plutôt sur la confrontation des mémoires : celle des élèves, celle de leur famille, et celle des habitants de leur ville. Les élèves ont élaboré une enquête sur la perception des attentats par les habitants d'Aulnay, ils ont interrogé 312 personnes et analysé leurs données chiffrées. Leurs questions portaient sur les impressions ressenties par les Aulnaysiens, sur leurs opinions quant aux auteurs des attentats et sur les conséquences nationales et internationales de l'événement. Ils ont ainsi découvert que les affirmations qui étaient considérées comme des évidences par nombre d'entre eux dans la classe n'étaient pas partagées par tous (le complot des « Illuminatis » notamment).

\section{Extrait d'une enquête réalisée par les élèves avec leur professeur d'Histoire}

Cette enquête a été réalisée par les élèves de la Première STG2 du lycée Voillaume avec leur professeur d'Histoire, Mme Abiven.

\section{Vous souvenez-vous de ce que vous faisiez lorsque vous avez appris les attentats du 11 septembre?}

Nous pouvons constater que parmi les personnes âgées de plus de 25 ans, $72 \%$ se souviennent précisément de ce qu'elles faisaient lors des attentats, ce qui confirme l'impact très important de ces événements sur la population ; parmi les jeunes de moins de 25 ans, un sur deux seulement s'en souvient. En toute logique, les jeunes ont été moins marqués par cet événement. (Stéphanie)

\section{Qu'avez-vous ressenti en réalisant ce qui s'était passé ?}

Le sentiment qui est revenu chez $28,5 \%$ des personnes interrogées est celui de la surprise, en partie car il s'agit des États-Unis, la première puissance économique et militaire mondiale.

$23,5 \%$ ont évoqué un sentiment de peur. Leur principale crainte était que cela arrive en France.

$22,1 \%$ ont éprouvé de la tristesse. Lors de l'attentat du 11 septembre 2001 on compte un total d'environ 2996 victimes. Certains Aulnaysiens se sont réjouis de cet événement, parfois en référence au conflit israélo-palestinien, mais ils sont très minoritaires, environ $1 \%$ seulement.

$12,2 \%$ des Aulnaysiens se sont montrés indifférents face aux attentats du 11 septembre 2001. La majorité d'entre eux sont des jeunes de moins de 25 ans, dont les $3 / 4$ sont de sexe féminin. Certains habitants d'Aulnay-sous-Bois justifient cela par le fait qu'il y a d'autres misères dans le monde que celle-ci. Lors de cette enquête on a eu l'occasion d'entendre : " Cessez de parler du 11 septembre 2011. Les Américains n'ont pas le monopole de la souffrance ». (Khalid, Eloïse et Karima)

\section{D'après vous, qui a commis ces attentats?}

Près de la moitié des Aulnaysiens pense que ce sont des terroristes islamistes qui ont commis ces attentats, conformément à la version officielle. Ben Laden, à la tête du réseau AlQaïda, a en effet revendiqué ces attentats. Néanmoins, une personne sur 4 estime que les Américains, plus précisément l'administration Bush, sont impliqués dans ces attentats, soit parce qu'ils les auraient commis eux-mêmes $(17 \%)$, soit parce qu'ils auraient participé à un complot avec Ben Laden (9\%). 7\% répondent que ce sont les Arabes et/ou les Musulmans en 
général qui seraient responsables, révélant ainsi un début de stigmatisation de ces populations. Quelques Aulnaysiens pensent étonnamment aux Irakiens, cela explique d'après eux pourquoi l'armée américaine a envahi l'Irak deux ans plus tard, en 2003. Au sein du lycée Voillaume, une rumeur court selon laquelle ce serait les illuminatis, une organisation secrète composée d'hommes puissants, qui auraient participé à cet attentat. Pourtant les enquêtes ont révélé que seule une toute petite minorité d'Aulnaysiens croit à cette rumeur. Enfin, concernant les personnes qui ne savent pas qui a commis ces attentats, on pourrait en déduire que certains ne s'intéressent pas à cet évènement, mais aussi et surtout qu'il reste de nombreuses «zones d'ombres » dans la compréhension de cet événement. (Franceline et Supitha)

\section{Quelle est selon vous la principale conséquence à l'échelle internationale de cet événement?}

Il faut d'abord souligner que $43,7 \%$ des personnes interrogées ont répondu qu'elles ne voyaient pas quelles avaient pu être les conséquences internationales de cet événement dans le monde. Si ces attentats ont énormément marqué les esprits, près d'un Aulnaysien sur deux n'a pas conscience de leur impact géopolitique. Parmi les personnes qui ont répondu, 60, soit $20 \%$, pensent aux guerres. 13 d'entre elles précisent la guerre en Irak qui est une intervention menée par les États-Unis depuis 2003. À l'inverse, 7 personnes seulement citent la guerre en Afghanistan alors que celle-ci a débuté seulement quelques semaines après les attentats. Les Aulnaysiens qui ont évoqué la montée de l'islamophobie dans le monde sont presque aussi nombreux que ceux qui ont pensé aux conflits internationaux. Les habitants d'Aulnay-sousBois, certainement très touchés par la crise économique actuelle, ont été assez nombreux (plus de $8 \%$ ) à croire que les attentats du 11 septembre 2001 en étaient à l'origine. Enfin, 5,5\% ont évoqué d'autres attentats; nous supposons qu'il s'agit des attentats de Londres en 2005 ou de Madrid en 2004. (Anne-Sophie, Vishal)

\section{Quelles ont été vos craintes?}

Nous constatons que la principale crainte des Aulnaysiens, hommes et femmes, tous âges confondus, est que des attentats se reproduisent ailleurs, surtout en France. Dans un second temps, les Aulnaysiens craignaient une troisième guerre mondiale. Dans les deux cas, ce sont des situations de grande violence qui ont été le plus redoutées. Enfin, 15\% des habitants d'Aulnay n'ont pas ressenti de craintes spéciales, peut-être se sont-ils sentis protégés car éloignés du continent américain. (Noémie et Silan)

Pour conclure, ces enquêtes nous ont permis de connaître l'avis des gens par rapport à cet événement et de pouvoir les comparer au nôtre. Leurs réponses étaient parfois surprenantes, par exemple quand les personnes nous répondaient qu'il s'agissait d'un complot entre Bush et Ben Laden, ou ceux qui nous parlaient des Illuminatis ou encore ceux qui pensent que les Arabes sont tous des terroristes! Ces enquêtes révèlent combien les habitants d'Aulnay-sousBois craignent que ces attentats aient pu stigmatiser les populations arabes et/ou musulmanes. (Anne-Sophie, Tarik, Stéphanie)

En cours de français, nous ne nous sommes pas livrés aux délices du commentaire de texte sur 11 septembre 2001. Les élèves ont abordé la pièce par la bande, en lisant d'abord une ou deux autres pièces de Michel Vinaver (Dissident il va sans dire et une partie de La Demande d'emploi), en observant les points de convergence ou les écarts entre ces textes et en en proposant une description chorale. Ils ont malgré tout écrit un "commentaire» de 11 septembre 2001 sous une forme détournée : celle du pastiche. La classe a pris appui sur le principe d'écriture qui gouverne la pièce (sélection de phrases trouvées dans la presse ou sur internet, montage placé sous le signe de la collision, choix du vers libre ou du verset, chœur 
en anglais) et elle l'a envisagé comme une proposition ou comme une sorte de " machine à écrire ». Restait à choisir un événement contemporain d'importance internationale ; comme ce texte a été rédigé à l'occasion de la venue de Michel Vinaver dans le lycée, les hasards du calendrier ont voulu que ce soit la Révolution tunisienne. 14 janvier 2011, le texte qui a été rédigé collectivement par la classe, relève incontestablement de l'exercice scolaire; il a pourtant pris une dimension nouvelle lors de la lecture que les élèves en ont faite pour Michel Vinaver. Son intensité venait tout à la fois de son caractère polyphonique, de la force du chœur et de la prononciation arabe que certains élèves se sont autorisée pour dire les noms des manifestants. Ce qui importe dans ce travail d'écriture, c'est qu'une forme inventée par Michel Vinaver pour dire le 11 septembre 2001 a pu être reprise pour parler de la révolution tunisienne, en une association étroite du politique et du poétique.

\section{Le cours : lieu du retour}

La préparation et le retour sur les rencontres, sur les sorties et sur les ateliers de pratique ont également été largement abordés en cours. Des écrits ont été systématiquement demandés aux élèves moins sur leurs lectures que sur leurs expériences théâtrales en tant que spectateurs ou en tant qu'acteurs. Ce que cette année de projet nous aura donc peu à peu appris, à nous enseignants, c'est à faire évoluer nos demandes, nos consignes, nos questions, afin de conduire les élèves à dépasser les jugements souvent un peu expéditifs (j'aime/je n'aime pas) pour tenter de formuler des impressions, des sensations, des états de conscience à la fois intenses et fugaces, de ces moments qu'il est si difficile de définir quelques jours après les avoir ressentis et qu'il faut mettre en mots très vite, avant que ne retombe le discours stéréotypé (l'inévitable « le théâtre, ça aide à être moins timide »).

Ces petits textes rédigés par les élèves rejoignent parfois certaines déclarations de Pascal Rambert par leur manière d'insister sur l'incandescence du moment théâtral ; mais, à la différence des comédiens décrits avec humour par Pascal Rambert, ils éprouvent cette incandescence en eux-mêmes, dans leur propre corps, et n'ont pas encore l'ambition de la projeter en direction du public. Là où les acteurs veulent « carboniser (...) leur auditoire sur place » ou « veulent voir de la fumée sortir des oreilles de ceux qui les écoutent ${ }^{2}$ », nos élèves disent leur expérience de l'intensité du moment théâtral en eux, leur impression - pour des élèves dits « de banlieue » qui sont toujours et avant tout définis par leur appartenance à la périphérie - d'être enfin au centre :

\footnotetext{
Ce qui est intense sur scène, ce sont les gens qui vous regardent sans cligner des yeux et qui ne vous lâchent pas du regard ; ils s'intéressent à nous. (Tahirou)

Sur scène, on raconte des choses à des personnes, on s'exprime d'une manière très préparée et vraiment intense. (Ibrahim)

Ce qui est intense sur scène, c'est l'écoute et la concentration du groupe. Quand je dis mon texte, c'est comme s'il me dominait. (Djénéba)

Ce qui est intense, c'est lorsqu'on est concentré et qu'on ne fait qu'un avec son personnage. On voit, on ressent l'émotion qu'on dégage lorsqu'on joue. (Maive).
}

Cette parole des élèves, quelle que soit sa forme, de la plus spontanée à la plus travaillée, a été mise en valeur comme jamais auparavant dans le cadre de ce projet : elle apparaît très largement sur la page Facebook dédiée à "D'un 11 septembre à l'autre ", sur le site de Citoyenneté Jeunesse, sur les blogs des lycées Jean Renoir et Evariste Galois ou sur la webradio du lycée Voillaume. Elle est même apparue sur scène à l'occasion d'une

\footnotetext{
${ }^{2}$ Pascal RAMBERT, L'Art du théâtre, Toute la vie suivi de L'Art du théâtre, Les Solitaires intempestifs, 2007.
} 
présentation du travail des élèves devant leurs familles au mois d'avril. Au cours de cette présentation, les comédiens ont fait la surprise aux élèves de lire sur le plateau, face au public, des extraits des textes qu'ils avaient écrits tout au long de l'année : des remarques sur des spectacles, des impressions ressenties sur le plateau, des définitions du théâtre, des questions préparées pour Michel Vinaver, des messages laissés sur le « Mur» de la page Facebook, ou des monologues intérieurs. Ce moment a institué une sorte d'échange entre les lycéens qui portaient le texte de Michel Vinaver et les comédiens qui métamorphosaient ces mots d'élèves en leur donnant droit de cité sur le plateau.

\section{«Et maintenant et maintenant et maintenant »}

Et maintenant, où en sommes-nous ? Où en sont nos élèves ? Qu'y a-t-il de changé en eux, entre eux, entre eux et nous leurs enseignants, entre eux et l'école, entre eux et le théâtre, entre eux et le regard qu'ils posent sur le monde ?

Ce qui semble incontestable, c'est l'écart qui sépare la fin du début de l'année dans leur approche du 11 septembre, non pas tant en termes d'analyse, mais dans le fait que l'évocation de cet événement soit absolument dépassionnée. Scène vue, dans le RER ou le bus, entre des élèves en route pour une journée de répétition sur 11 septembre 2001 : deux d'entre eux répètent leur texte au milieu des voyageurs : «moi je fais Bush », " moi je fais Ben Laden » : «Pour faire disparaître le mal / Le nom de l'opération militaire d'aujourd'hui / Je jure devant Dieu / Est Liberté Immuable / Que 1'Amérique ne vivra pas en paix... ». Les élèves étaient d'un naturel absolu ; moi, leur professeur, j'éprouvais une indéniable gêne à entendre ce texte prononcé haut et clair dans les transports en commun, au milieu d'un public non prévenu. Il est difficile de dire ce qui a rendu possible cette distance, ce naturel, cette familiarité : le passage par la fiction, la longue fréquentation du texte, les rencontres avec des intervenants ? Indéniablement pourtant le fait de jouer ce texte (un de ces textes qui « donnent des nouvelles du monde » selon le mot de Claude Régy), de le travailler, de le dire en public, de jouer un rôle, de changer de rôle, a modifié leur rapport à l'événement. L'an prochain, en Terminale, les élèves aborderont l'événement en cours d'Histoire. C'est à ce moment-là sans doute que nous pourrons mesurer avec le plus de justesse ce qui s'est modifié non seulement dans leur positionnement à l'égard de l'événement, mais aussi dans l'analyse qu'ils en font. Pour l'heure, l'enthousiasme à l'idée des représentations à venir l'emporte sur toute autre considération (ainsi de Sabrina qui a commenté en ces termes l'annonce de la mort de Ben Laden : « Cela fera sûrement de la publicité à notre projet ! »).

Bien sûr, quelque chose s'est aussi modifié dans leur manière de faire face à un public. Fruit du travail de plateau, du travail sur le corps, sur le regard, sur le geste, sur la voix, sur l'adresse, ce déplacement est aussi intimement lié à la modification de l'image que ces élèves ont d'eux-mêmes, à la conscience qu'ils ont désormais d'être vus, observés, à l'idée qu'un regard sera posé sur ce qu'ils auront fait, écrit, produit, inventé. De fait, ce projet les a sans cesse confrontés au regard des autres, sous des formes très différentes : regard porté sur leurs propositions en atelier, sur leur présence sur le plateau, instants saisis par les photos, les films, commentaires sur leurs textes, sur leurs messages, etc. Si leur présence sur le plateau est vouée à avoir le caractère éphémère d'une création théâtrale, on ne peut s'empêcher d'entendre le dialogue qui s'est établi entre ces jeunes gens et le théâtre, aussi bien en tant que comédiens qu'en tant que spectateurs. Et quand on voit le nombre de textes écrits cette année par les élèves sur leur expérience du théâtre ou sur les pièces qu'ils ont vues, on songe au 
propos de Bernard Dort qui voit dans la parole du public sur la pièce un élément constitutif du « grand jeu théâtral » et l'on peut à bon droit espérer que ce dialogue se poursuive : «Parler du théâtre, c'est précisément continuer le jeu, prolonger ce dialogue entre une œuvre et notre société qui, de la scène, a gagné la salle ${ }^{3} »$.

À propos de l'auteur :

Marie-Laure Basuyaux est agrégée de Lettres Modernes, docteur en Littérature française (Ecrire après. Les récits lazaréens de Jean Cayrol, 2005), membre associé de l'équipe Littératures françaises du XXe siècle de l'Université Paris IV-Sorbonne et professeur au lycée Voillaume d'Aulnay-sous-Bois. Son essai sur la littérature lazaréenne, Témoigner clandestinement (2009) est édité chez Garnier Classiques, dans la collection «Etudes de littérature des XXe et XXIe siècles ».

Pour citer ce document :

Marie-Laure Basuyaux, «Le cours, lieu du détour et du retour», Agôn [En ligne], Dossiers, $\mathrm{HS} \mathrm{n}^{\circ} 1$ : Mettre en scène l'événement, D'un 11 septembre à l'autre, mis à jour le : 06/09/2011, URL : http://agon.ens-lyon.fr/index.php?id=1794.

\footnotetext{
${ }^{3}$ Bernard Dort, Théâtres - Essais, « Avant-propos », Paris, Seuil, 1986, p. 13.
} 